\title{
Introduction to Group-Structured-Epidemic Model
}

\author{
Samaherni $\operatorname{Dias}^{1}\left[\right.$ [D $\cdot$ Kurios Queiroz ${ }^{1} \cdot$ Aldayr Araujo $^{1}$ \\ Received: 27 March 2021 / Revised: 27 July 2021 / Accepted: 20 September 2021 / Published online: 31 October 2021 \\ (c) Brazilian Society for Automatics-SBA 2021
}

\begin{abstract}
The spread of an infectious disease in a population is a random process when considering a small group of individuals. However, to a great group of individuals, the use of deterministic behavior is better. Based on these facts, in the literature, there were proposed stochastic and deterministic epidemic models. This work proposes a mixed compartmental epidemic model that allows stratifying the population into groups, considers demographic and environmental variability, presents an approximation to stochastic effects, and contemplates the network effects. The proposed model has a compact form to assist in the synthesis of the control law and parameters estimation strategies. Its objective is to overcome the difficulties encountered when used purely deterministic or purely stochastic models. In the end, to detail and verify the functioning of the proposed model, we present a set of flowcharts and simulations.
\end{abstract}

Keywords COVID-19 $\cdot$ Epidemic disease $\cdot$ Deterministic model $\cdot$ Stochastic model

\section{Introduction}

An infectious disease is a clinically evident illness resulting from the presence of a pathogenic microbial agent. This agent can be bacterial, viral, fungal, parasitic, or toxic proteins, called prions (Martcheva 2015). Transmission of infectious diseases may occur through a variety of pathways. According to the means of transmission, we can classify the infectious diseases as follows: person-to-person, food-and-waterborne diseases, airborne transmission, vector-borne diseases, and vertical transmission.

To study the epidemic disease dissemination, we can use mathematical models, called epidemic models. Generally, these models divide the population into classes. The transition of individuals between classes occurs in two forms: deterministic or stochastic. In the deterministic case, the transition occurs according to the mean value of transmission rate between individuals from different classes. In the stochastic case, instead of the mean value, it uses a probabilistic distribution. In addition to deterministic and stochastic, we can classify the epidemic models as follows: linear/nonlinear, static/dynamic, discrete/continuous.

Samaherni Dias

sama@laci.ufrn.br

1 Laboratory of Automation, Control, and Instrumentation, Department of Electrical Engineering, Federal University of Rio Grande do Norte, Natal, RN, Brazil
In the history of epidemic disease models, we can highlight the works of McKendrick (1925) and Kermack et al. (1927). In the first (McKendrick 1925), the author proposes a stochastic model and, in the second (Kermack et al. 1927), the authors propose a deterministic model called SIR. The name SIR is due to the classification adopted by the authors. In the SIR model, we divide the population into three classes: susceptible, infectious, or recovered. The SIR model is a special type of model called the compartmental model because each letter refers to a "compartment" in which an individual can reside. Each individual can reside in exactly one compartment and can move from one compartment to another (Martcheva 2015).

In the last 100 years, researchers are developing new stochastic and deterministic models. We can find the most traditional epidemics models detailed in the books of Daley and Gani (1999) and Martcheva (2015). Nowadays, the study of epidemics models keeps in trending top with several recent publications. To deterministic models, we can highlight Zakary et al. (2017a), Blackwood and Childs (2018), Li et al. (2020), Davies et al. (2020), Prem et al. (2020), Hilton and Keeling (2019), and to stochastic models, we can highlight Newman (2002), Moreno et al. (2002), Britton (2010), Black and McKane (2012), Artalejo et al. (2013), Ortega et al. (2013), Pastor-Satorras et al. (2015), Zhang et al. (2015), López-García (2016), Nowzari et al. (2016), Allen (2017), Jiang and Zhou (2018), Wang and Jia (2019), Kucharski et al. 
(2020). Being to the stochastic model, the works considering complex networks are growing.

The spread of an infectious disease is a random process in a small group of individuals. When the group of individuals is very large, it is customary to represent the infection process deterministically. Thus, deterministic models are unsuitable for small populations, and stochastic models do not present satisfactory results for a larger population (Daley and Gani 1999). In Allen and Burgin (2000), we find a comparison of deterministic and stochastic SIS and SIR models in discrete time.

Stochastic modeling of epidemics is important when the number of infectious individuals is small or when the variability in transmission, recovery, births, deaths, or the environment impacts the epidemic outcome (Allen 2017). There are two variabilities: one associated with individual dynamics and the other with the environment. The first, called demographic variability, results from individuals dynamics such as transmission, recovery, births, or deaths. The second, called environmental variability, results from conditions related to terrestrial or aquatic settings.

Mixed models were proposed to overcome the difficulties related to purely stochastic or deterministic models. Within the fully mixed models class, much elaboration is possible, particularly concerning the effects of age structure in population and population turnover. The crucial element that all such models lack is network topology. A given infective individual does not have an equal probability of infecting all others (Newman 2002). To the model describe some specifics phenomena appropriately, it is important to consider the topology of social contact networks. For example, the crowding phenomenon occurs when there is a significant number of infected individuals in a community preventing the fast spreading of the disease (Zhang et al. 2015).

Roberts et al. (2015) show nine challenges for deterministic epidemic models. The authors indicate the models have to include multi-strain, time-varying infectivity, and superinfection. They also consider it necessary to improve the epidemic spatial models (large regions), explore the interaction with non-communicable diseases, and develop robust deterministic approximations of stochastic models.

According to Bedford et al. (2019), the epidemics will become more frequent, complex, and harder to prevent and contain. The authors claim that we need to develop new approaches to epidemic prevention and response. Bedford et al. (2019) assign these changes to the demographic transition, inequalities, climate change, agricultural and forestry practices, and accessible travels around the world. In other words, non-biological aspects are affecting the epidemics. The recent publications include non-biological aspects in the models. Some of these works (Davies et al. 2020; Duan et al. 2020; Canabarro et al. 2020) consider age structure, others consider multi-regions (Zakary et al. 2017a,b), and some consider multiple factors (Hilton and Keeling 2019; Acemoglu et al. 2020; Dias et al. 2021a, b, 2020). Resuming, we need to consider social aspects to achieve a better representation of an epidemic disease outbreak.

Another important aspect of epidemic diseases models is the time-varying parameters. Classical models may not capture the dynamic transmission and removal processes (Hong and $\mathrm{Li} 2020$ ). These models do not describe the various phases of the epidemic disease. Use time-varying parameters allows us to capture possible changes in the epidemic behavior (Calafiore et al. 2020). Treatments, mutations, and containment measures enforced by authorities can change the characteristics of an epidemic disease (Calafiore et al. 2020). According to Sabino et al. (2021), the mutations may have caused a resurgence of COVID-19 cases in Manaus, Brazil. The authors observe that a new lineage circulating in the second wave had higher inherent transmissibility than pre-existing lineages circulating in Manaus. Finally, the estimation of time-varying parameters can help the decisionmakers evaluate the efficiency of adopted measurements. During the coronavirus outbreak (COVID-19) in Wuhan, You et al. (2020) estimated the reproduction number time-varying of the disease to investigate the containment measures carried out by the Chinese government.

Based on the main challenges, the researchers proposed several models. Each one of them with specific characteristics and to be applied in particular epidemic behavior. The difficulty in proposing an ideal model produces new models periodically. For each new model, the researchers introduce a set of new considerations. At this point, we can conclude that proposing a model considering a specific outbreak or to a determined social aspect is so restrictive that we need to develop a versatile model for wide applications. This model has to show a good description of several epidemic diseases outbreaks.

We believe that the new approaches to epidemic prevention and response need a paradigm shift, which means a new and completely different form to propose a solution to this problem. Proposing models to more frequent and complex epidemic diseases will be an activity to the Artificial Intelligences (AI). In the actual stage and considering the future perspectives, we have enough data about the population to AI techniques, following specific rules, defines the better model to describe an epidemic disease during the outbreak. In this work, we propose a new schematic to model epidemic diseases. We are developing this schematic, to in the future, it works as a set of rules to AI techniques. This schematic we call group-structured-epidemic model (GSEM), and we can use it to describe any other compartmental epidemic model. Thus, the GSEM is a nonlinear model that can describe an epidemic disease with any compartment number and independent relationship between biological and populational aspects. 
Then, the proposed model will be versatile and compact. To be versatile, we propose a new relationship organization that implies the full decoupling of the epidemic's description aspects. We consider it compact because all epidemic descriptions are parameters of the matrices.

Contributions. This work proposes a nonlinear, dynamic, and continuous new mixed compartmental epidemic model that stratifies the populational aspects into groups. It considers the social contact network effects, the stochastic effects, the demographic and environmental variabilities, and it will be compact to help in the strategies of control and parameters estimation.

We organized the papers as follows: In Sect. 2, the deterministic epidemic SIR model is introduced. In Sect. 3, we will present the proposed model. Section 4 contains some numerical simulations of the theory. In the end, Sect. 5 provides some concluding comments and some challenges for future works.

\section{Traditional Models}

The introduction to epidemic modeling is usually made through one of the first epidemic models proposed by Kermack et al. (1927), a model known as the SIR epidemic model (Martcheva 2015). In that work, the authors propose a mathematical model to describe an epidemic disease when considering the population divided into three different classes (susceptible, infectious, recovered). To SIR model, we have to do two considerations: the infected individuals can transmit the disease; the number of individuals in the population is constant $(p)$. Because of the last consideration, we can apply the SIR model, proposed in 1927, in short period epidemic outbreaks (few months).

When elaborating a mathematical model to a biological event, the first step is to describe it adequately. Thus, to the SIR model, the transition between classes occurs as follows. When a susceptible individual enters into contact with an infected individual, there is a certain probability to the susceptible individual becomes infected and moves from the susceptible class into the infected class. Every infected individual, after a period $T_{r}$, moves from the infected class into the recovered class.

Consider $s(t)$ the number of susceptible individuals, $i(t)$ the number of infected individuals, $r(t)$ the number of recovered individuals, and $c(t)$, called incidence, the number of individuals who become infected per unit of time. The incidence is

$$
c(t)=\beta(t) i(t) \frac{s(t)}{p}=\tau(t) \kappa(t) i(t) \frac{s(t)}{p},
$$

where $\beta(t)$ is a coefficient proportional to the disease transmission rate. $\tau(t)$ is the probability of contact between a susceptible individual and an infected individual results in transmission. $\kappa(t)$ is the contact number that an infected individual has per unit of time. The term $s(t) / p$ is a probability that occurs contact between an infected individual and a susceptible individual.

The SIR model consists of a system of three differential equations:

$$
\begin{aligned}
\frac{\mathrm{d} s(t)}{\mathrm{d} t} & =-\beta(t) i(t) \frac{s(t)}{p}, \\
\frac{\mathrm{d} i(t)}{\mathrm{d} t} & =\beta(t) i(t) \frac{s(t)}{p}-\gamma(t) i(t), \\
\frac{\mathrm{d} r(t)}{\mathrm{d} t} & =\gamma(t) i(t),
\end{aligned}
$$

where $\gamma(t)$ is the recovery rate, and it is the result of the inverse of infection time $\left(T_{r}\right)^{-1}$.

The fundamental characteristics of the model (2) to describe an epidemic outbreak are:

C1. the initial condition of $s(t)$ is $s(0)=s_{0}>0$;

C2. the initial condition of $i(t)$ is $i(0)=i_{0}>0$;

C3. the value of the number of contacts that an infected individual has per unit time is $\kappa(t)>0$;

C4. the probability that a contact with susceptible individual results in transmission is $\tau(t)>0$;

C5. the recovery rate is $\gamma(t)>0$;

In the traditional SIR model (2), the values of $\beta(t)$ and $\gamma(t)$ are constants. They represent the mean values of these parameters to a well-mixed population.

About the SIR model, we have to highlight the basic reproduction number of the disease

$$
R_{0}(t)=\frac{\beta}{\gamma}
$$

It gives the number of secondary cases one infectious individual will produce in a population consisting only of susceptible individuals (Martcheva 2015).

Other models, as SIRS and SEIR ${ }^{1}$, derive from the SIR model (see Fig. 1). The variations of the traditional SIR model we call of SIR-type models. SIR-type models are a set of possible configurations. For example, the models can consider demographic variabilities, vaccination, stratification, vectorborne, among others.

We also can represent stochastic models by the flowchart of Fig. 1. In this case, we use the stochastic process in the transitions of classes, at all or part of them. To the models based on social network contact, we use the theory of

\footnotetext{
${ }^{1}$ The letter E represents the exposed individuals.
} 


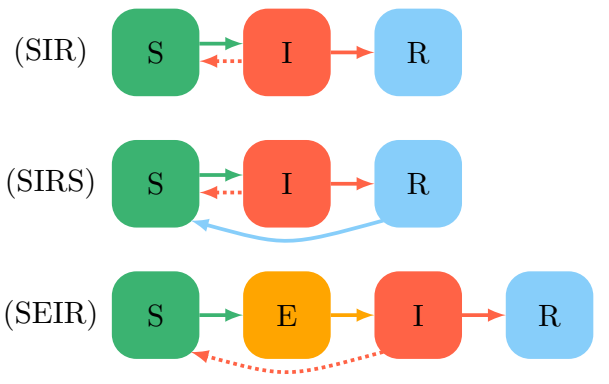

Fig. 1 Classes flowchart to SIR-type models

complex networks to determine the relationship between the individuals in any class.

\section{Proposed Model}

In this work, we propose a mixed compartmental epidemic model that stratifies the populational aspects into groups. We consider it mixed because it is a deterministic model that considers the social contact network effects and the stochastic effects. To our model, we divide the population into classes. And we can divide each class into groups. The change of individuals between classes, we call transition, and between groups, we call flux. The flux is a new concept introduced in this work to a better description of the populational aspects.

To help in the understanding of the proposed model, we develop auxiliary models. Each new model will work as a step until the complete model. These intermediary models allow the reader have a better knowledge of the concepts defined in the proposed model.

The first auxiliary model is the group-structured-SIR model (GSIR). This model divides the population into three classes (S, I, R) and stratifies each class into groups. Each group can represent a specific characteristic, per examples, age, location, gender, among others.

The idea behind the proposed model is to fragment the heterogeneous population into homogenous groups. To each homogenous group applies a deterministic model and all dynamic involved in the relationship between groups.

\subsection{Description of Notations}

From now until the end, we consider all bold capital letters are matrix, and we can represent it by $\boldsymbol{A}$ or $(a)_{z j}$. We represent the elements of $\boldsymbol{A}$ matrix by $a_{(z, j)}$. All bold tiny letters are vectors.

We consider all variables below a bar are constants, and the other variables are time variant. Consider the time derivative by $\frac{\mathrm{d} \boldsymbol{q}(t)}{\mathrm{d} t}={ }_{\partial} \boldsymbol{q}$,

where the derivative of a vector is the derivative of each element of this vector. Consider the Hadamard operators $\odot$, $\oslash,(.)^{\circ-1}$ as point-wise multiplication, point-wise division, and the point-wise inverse of vector elements, respectively.

\subsection{Group-Structured-SIR Model (GSIR)}

The first auxiliary model we propose is GSIR. Consider an epidemic model to the population organized into three classes $(\mathrm{S}, \mathrm{I}, \mathrm{R})$ and each class with $n_{g}$ groups. The vectors $s \in \mathfrak{R}^{n_{g}}$, $\boldsymbol{i} \in \mathfrak{R}^{n_{g}}, \boldsymbol{r} \in \mathfrak{R}^{n_{g}}$ contain the number of individuals of each class organized by groups. The $\boldsymbol{s}, \boldsymbol{i}, \boldsymbol{r}$ vectors represent the susceptible, infected and, recovered individuals, respectively. The GSIR model is a system with three derivative equations of three vectors and detailed by

$$
\begin{aligned}
{ }_{\partial} \boldsymbol{s} & =-\left(\boldsymbol{B}_{s} \cdot \boldsymbol{i}\right) \odot\left(\boldsymbol{s} \oslash \boldsymbol{p}_{g}\right)+\boldsymbol{\phi}_{s}, \\
{ }_{\partial} \boldsymbol{i} & =\left(\boldsymbol{B}_{s} \cdot \boldsymbol{i}\right) \odot\left(\boldsymbol{s} \oslash \boldsymbol{p}_{g}\right)-\boldsymbol{\Gamma}_{i} \cdot \boldsymbol{i}+\boldsymbol{\phi}_{i}, \\
{ }_{\partial} \boldsymbol{r} & =\boldsymbol{\Gamma}_{i} \cdot \boldsymbol{i}+\boldsymbol{\phi}_{r},
\end{aligned}
$$

where $\boldsymbol{\phi}_{s} \in \mathfrak{R}^{n_{g}}, \boldsymbol{\phi}_{i} \in \mathfrak{R}^{n_{g}}, \boldsymbol{\phi}_{r} \in \mathfrak{R}^{n_{g}}$ are the vectors with the flux of individuals between groups in the same class, where $\boldsymbol{\phi}_{s}, \boldsymbol{\phi}_{i}, \boldsymbol{\phi}_{r}$ are to susceptible, infected and recovered individuals, respectively. $\boldsymbol{B}_{s} \in \mathfrak{R}^{n_{g} \times n_{g}}$ is the matrix with coefficients of the disease transmission rate, and $\boldsymbol{\Gamma}_{i} \in \Re^{n_{g} \times n_{g}}$ is the matrix with the recovered rate of each group in your main diagonal. The last one is

$\boldsymbol{p}_{g}=\boldsymbol{s}+\boldsymbol{i}+\boldsymbol{r}$,

which is the vector with the population divided by groups.

The matrix with coefficients of the disease transmission rate is

$\boldsymbol{B}_{s}=\boldsymbol{T}_{s} \odot \boldsymbol{K}_{s}$,

where $\boldsymbol{T}_{s}=\left(\tau_{s}\right)_{z j} \in \Re^{n_{g} \times n_{g}}$ represents the matrix with the probability of contact between a susceptible individual of group $j$ and an infected individual of group $z$ results in transmission. The matrix $\boldsymbol{K}_{s}=\left(\kappa_{S}\right)_{z j} \in \Re^{n_{g} \times n_{g}}$ represents the social network and the contact number that an infected individual has per unit of time. In other words, it defines the contact number of infected individuals of group $j$ has, per unit of time, with susceptible individuals of group $z$.

We can calculate the vector with the flux between the groups in four different ways: 
$\boldsymbol{\phi}_{q}=\boldsymbol{\Omega}_{q} \cdot \mathbf{1}_{n_{g} \times 1}$,

$\boldsymbol{\phi}_{q}=\left(\boldsymbol{\Omega}_{q(A \rightarrow B)}+\boldsymbol{\Omega}_{q(B \rightarrow A)}\right) \cdot \mathbf{1}_{n_{g} \times 1}$,

$\boldsymbol{\phi}_{q}=\left(\boldsymbol{\Lambda}_{q} \odot\left(\boldsymbol{p}_{g}^{\circ-1} \boldsymbol{p}_{g}^{T}\right)\right) \cdot \boldsymbol{q}$,

$\boldsymbol{\phi}_{q}=\left(\left(\boldsymbol{\Lambda}_{q(A \rightarrow B)}+\boldsymbol{\Lambda}_{q(B \rightarrow A)}\right) \odot\left(\boldsymbol{p}_{g}^{\circ-1} \boldsymbol{p}_{g}^{T}\right)\right) \cdot \boldsymbol{q}$,

$q=s, i, r$,

where $\mathbf{1}_{z \times j} \in \Re^{z \times j}$ represents the matrix with all elements equals to one.

The first two forms consider the number of individuals, and to them, matrix $\boldsymbol{\Omega}_{q}$ represents the balance of the number of individuals that changed the group at a time interval. The matrices $\boldsymbol{\Omega}_{q(A \rightarrow B)}, \boldsymbol{\Omega}_{q(B \rightarrow A)}$ contains the number of individuals that move from group A to group B and from group B to group A, respectively.

The last two forms consider the percentile of individuals, and to them, matrix $\boldsymbol{\Lambda}_{q}$ represents the balance of the percentual of individuals that changed the group at a time interval. The matrices $\boldsymbol{\Lambda}_{q(B \rightarrow A)}, \boldsymbol{\Lambda}_{q(B \rightarrow A)}$ contain the percentiles of individuals that move from group A to group B and from group B to group A, respectively. The term $\left(\boldsymbol{p}_{g}^{\circ-1} \boldsymbol{p}_{g}^{T}\right)$ is to adjust the percentiles according to the population of each group.

Still about (7), the elements $\omega_{q(z, j)}, \lambda_{q(z, j)}$ of the matrices $\boldsymbol{\Omega}_{q}, \boldsymbol{\Omega}_{q(A \rightarrow B)}, \boldsymbol{\Omega}_{q(B \rightarrow A)}$ and $\boldsymbol{\Lambda}_{q}, \boldsymbol{\Lambda}_{q(A \rightarrow B)}, \boldsymbol{\Lambda}_{q(B \rightarrow A)}$, respectively, have the following logic: when $z=j$ represents the demographic flux; when $z \neq j$ represents the flux between the groups and the elements $(.)_{z, j},(.)_{j, z}$ has the same values with opposite signals. The matrix $\boldsymbol{\Gamma}_{i}$ is responsible for the transition between classes that do not depend on the interaction between classes. And their elements $\gamma_{i(z, j)}$ are the transition rates, when $z=j$, and are null when $z \neq j$.

The GSIR model adapts the traditional SIR model to a population stratified in any number of groups. The objective is to reach better results of SIR models when applied to heterogeneous populations. The groups should divide the population according to similar characteristics. Thus, in each group, we have a more homogenous population, and we can adopt mean values to represents the biological aspects of this population.

When we apply the traditional SIR model to a non-wellmixed population, the model parameters become nonlinear, time-varying, and complex. These parameters are so complex that estimate them is very complicated. Instead of considering complex nonlinear time-variant parameters, the GSIR model considers a more sophisticated organization to the population.

\section{Example 1: GSIR model to stratify the population into two groups.}

To show an application of the GSIR model, in Fig. 2, we have a flowchart to describe the proposed example that stratifies the population into two groups with flux between groups and without demographic variabilities.

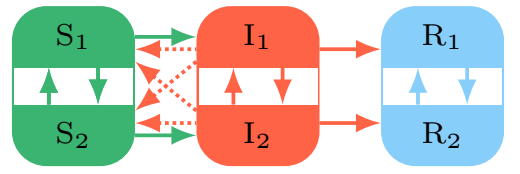

Fig. 2 Flowchart of the GSIR model applied to a population stratified into two groups with flux between groups and without demographic variabilities

According to Fig. 2, we have the population divided into three classes $(\boldsymbol{s}, \boldsymbol{i}, \boldsymbol{r}) \in \mathfrak{R}^{n_{g}}$, stratified into two groups $\left(n_{g}=\right.$ 2), with flux considering percentiles $\left(\boldsymbol{\Lambda}_{s}, \boldsymbol{\Lambda}_{i}, \boldsymbol{\Lambda}_{r}\right)$ and without demographic variabilities $\left(\forall z=j \Rightarrow \lambda_{q(z, j)}=0\right)$. In this case, we have

$$
\begin{aligned}
\boldsymbol{T}_{s} & =\left[\begin{array}{ll}
\tau_{s(1,1)} & \tau_{s(1,2)} \\
\tau_{s(2,1)} & \tau_{s(2,2)}
\end{array}\right], \quad \boldsymbol{K}_{s}=\left[\begin{array}{ll}
\kappa_{s(1,1)} & \kappa_{s(1,2)} \\
\kappa_{s(2,1)} & \kappa_{s}(2,2)
\end{array}\right], \\
\boldsymbol{\Gamma}_{i} & =\left[\begin{array}{cc}
\gamma_{i(1,1)} & 0 \\
0 & \gamma_{i(2,2)}
\end{array}\right], \quad \boldsymbol{\Lambda}_{s}=\left[\begin{array}{cc}
0 & \lambda_{s(1,2)} \\
-\lambda_{s(2,1)} & 0
\end{array}\right], \\
\boldsymbol{\Lambda}_{i} & =\left[\begin{array}{cc}
0 & \lambda_{i(1,2)} \\
-\lambda_{i(2,1)} & 0
\end{array}\right], \quad \boldsymbol{\Lambda}_{r}=\left[\begin{array}{cc}
0 & \lambda_{r(1,2)} \\
-\lambda_{r(2,1)} & 0
\end{array}\right],
\end{aligned}
$$

where the elements of the matrices $\boldsymbol{T}_{s}, \boldsymbol{K}_{s}, \boldsymbol{\Gamma}_{i}$ are nonnegative real numbers $\left(\Re_{+}\right)$, and all matrices elements are time variants.

\subsection{Group-Structured-SEIR Model (GSEIR)}

For many diseases, the infected individuals do not become immediately infectious. The pathogen needs time to replicate and establish itself in the new host. The time during which an individual is infected but not yet infectious is called the latent period. We note that the incubation period of a pathogen is the period between infection and onset of symptoms. The lengths of the latent and incubation periods do not necessarily coincide (Martcheva 2015).

The latent period usually follows the susceptible stage. As an additional compartment in epidemiological models, the latent period, also called the exposed period, is denoted by $\mathrm{E}$. If we introduce a latent period in the SIR model, the resulting model is a SEIR model.

The second auxiliary model is the group-structured-SEIR model. The idea is the same as the GSIR model, that is, to define more homogeneous groups and to use deterministic models.

The GSEIR model considers the population organized into four classes (S, E, I, R) and each class with $n_{g}$ groups. The vectors $\boldsymbol{s} \in \mathfrak{R}^{n_{g}}, \boldsymbol{e} \in \mathfrak{R}^{n_{g}}, \boldsymbol{i} \in \mathfrak{R}^{n_{g}}, \boldsymbol{r} \in \mathfrak{R}^{n_{g}}$ contain the number of individuals of each class divided by groups. The $\boldsymbol{s}, \boldsymbol{e}, \boldsymbol{i}, \boldsymbol{r}$ vectors represent the susceptible, exposed, infected and, recovered individuals, respectively. The GSEIR model is a system with four derivative equations of four vectors and 


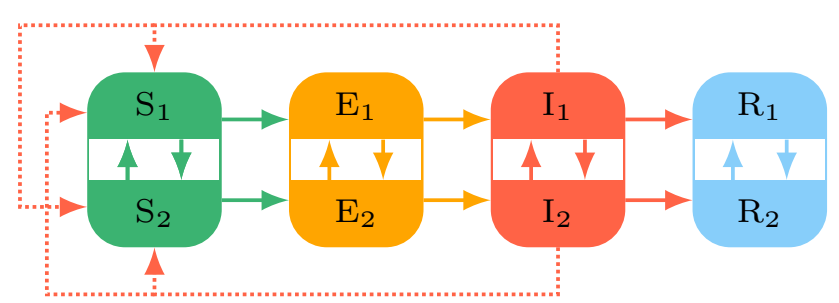

Fig. 3 Flowchart of the GSEIR model applied to a population stratified into two groups with flux between groups and without demographic variabilities

detailed by

$$
\begin{aligned}
{ }_{\partial} \boldsymbol{s} & =-\left(\boldsymbol{B}_{s} \cdot \boldsymbol{i}\right) \odot(\boldsymbol{s} \oslash \boldsymbol{p})+\boldsymbol{\phi}_{s}, \\
{ }_{\partial} \boldsymbol{e} & =\left(\boldsymbol{B}_{s} \cdot \boldsymbol{i}\right) \odot(\boldsymbol{s} \oslash \boldsymbol{p})-\boldsymbol{\Gamma}_{e} \cdot \boldsymbol{e}+\boldsymbol{\phi}_{e}, \\
{ }_{\partial} \boldsymbol{i} & =\boldsymbol{\Gamma}_{e} \cdot \boldsymbol{e}-\boldsymbol{\Gamma}_{i} \cdot \boldsymbol{i}+\boldsymbol{\phi}_{i}, \\
{ }_{\partial} \boldsymbol{r} & =\boldsymbol{\Gamma}_{i} \cdot \boldsymbol{i}+\boldsymbol{\phi}_{r},
\end{aligned}
$$

where

$p_{g}=s+e+i+r$.

The GSEIR model adapts the traditional SEIR model to a population stratified in any number of groups.

Example 2: GSEIR model to stratify the population into two groups.

To show an application of the GSEIR model, in Fig. 3, we have a flowchart to describe the proposed example that stratifies the population into two groups with flux between groups and without demographic variabilities.

If we follow the same procedure of Example 1, in the GSEIR case, we have to add two new matrices

$$
\boldsymbol{\Gamma}_{e}=\left[\begin{array}{cc}
\gamma_{e}(1,1) & 0 \\
0 & \gamma_{e(2,2)}
\end{array}\right] \quad \boldsymbol{\Lambda}_{e}=\left[\begin{array}{cc}
0 & \lambda_{e(1,2)} \\
-\lambda_{e(2,1)} & 0
\end{array}\right]
$$

The matrix $\boldsymbol{\Gamma}_{e}$ is the matrix with the latency rate of each group in your main diagonal.

\subsection{Proposed Group-Structured-Epidemic Model (GSEM)}

After analyzing the models (4), (8), it is possible to identify some common characteristics for both. The models organize the biological aspects in classes and the population division in groups. Individuals can change between different groups or classes.

The class changes occur by period or by the class interaction. The group changes result from the individual's desire or imposed by nature.

Here we propose a mixed compartmental epidemic model, called GSEM (group-structured-epidemic model), to describe any epidemic disease in which each individual can reside in exactly one compartment and move from one compartment to another. The compartment proposed has two qualifiers: a class and a group. The model is mixed because it is deterministic and considers stochastic effects. We can use the GSEM model to describe all SIR-type models.

Consider an epidemic disease with $n_{c}$ classes and $n_{g}$ groups described by

${ }_{\partial} \boldsymbol{x}=(\boldsymbol{B} \cdot \boldsymbol{x}) \odot(\boldsymbol{M} \cdot(\boldsymbol{x} \oslash \boldsymbol{p}))+\boldsymbol{\Gamma} \cdot \boldsymbol{x}+\boldsymbol{\phi}$,

where

$x^{T}=\left[\begin{array}{llll}x_{1} & x_{2} & \ldots & x_{n_{c}}\end{array}\right]$

is composed of $n_{c}$ vectors with the individuals stratified into groups given by

$\boldsymbol{x}_{z}^{T}=\left[\begin{array}{llll}x_{1} & x_{2} & \ldots & x_{n_{g}}\end{array}\right]$,

being $\boldsymbol{x} \in \Re^{n_{x}}$ the vector with all groups from all classes. $\boldsymbol{B} \in \Re^{n_{x} \times n_{x}}$ and $\boldsymbol{\Gamma} \in \Re^{n_{x} \times n_{x}}$ are the matrices with the rate of class interaction or noninteraction, respectively. $\boldsymbol{M} \in$ $\Re^{n_{x} \times n_{x}}$ is the matrix with the relationship between classes, $\phi \in \Re^{n_{x}}$ is the vector with the flux of individuals between groups and the demography (birth and death rates of each group), $n_{x}=n_{c} \cdot n_{g}$, and

$\boldsymbol{p}=\left[\begin{array}{c}\boldsymbol{p}_{g} \\ \boldsymbol{p}_{g} \\ \vdots \\ \boldsymbol{p}_{g}\end{array}\right] \in \Re^{n_{x}}, \quad \boldsymbol{p}_{g} \in \Re^{n_{g}}, \quad \boldsymbol{p}_{g}=\sum_{z=1}^{n_{c}} \boldsymbol{x}_{z}$

We calculate the $\boldsymbol{\phi}$ vector from the matrices $\boldsymbol{\Omega}_{q}, \boldsymbol{\Omega}_{q(A \rightarrow B)}$, $\boldsymbol{\Omega}_{q(B \rightarrow A)}, \boldsymbol{\Lambda}_{q}, \boldsymbol{\Lambda}_{q(A \rightarrow B)}, \boldsymbol{\Lambda}_{q(B \rightarrow A)}$ according to (7).

The fundamental characteristics of the model (10) to describe an epidemic outbreak are:

C6. The elements of the matrices $\boldsymbol{B}, \boldsymbol{M}, \boldsymbol{\Gamma}$ are nonnegative real numbers $\left(\Re_{+}\right)$;

C7. The elements of the vectors $\boldsymbol{x}, \boldsymbol{p}$ are nonnegative real numbers $\left(\Re_{+}\right)$;

C8. The initial condition of susceptible individuals, in at least one group, is greater than zero;

C9. The initial condition of infected individuals, in at least one group, is greater than zero;

A compact form to (10) is

${ }_{\partial} \boldsymbol{x}=\boldsymbol{F}_{n l}(\boldsymbol{x})+\boldsymbol{F}_{l} \cdot \boldsymbol{x}+\boldsymbol{\phi}$,

which represents the GSEM model by the separation of linear $\left(\boldsymbol{F}_{l}\right)$ and nonlinear $\left(\boldsymbol{F}_{n l}\right)$ relationships from the system. 
This compact form is important to become easier to develop new controller' laws, new parameters estimators, new applications of artificial intelligence, among other applications.

We add stochastic effects in the probability of contact between a susceptible individual and an infected individual results in transmission, in the social network and contact number that an infected individual has per unit of time, and in the flux of individuals.

For two firsts, we have

$\boldsymbol{B}_{s}=\left(\boldsymbol{T}_{s}+\widehat{\boldsymbol{T}}_{s}\right) \odot\left(\boldsymbol{K}_{s}+\widehat{\boldsymbol{K}}_{s}\right)$,

where $\widehat{\boldsymbol{T}}_{S} \in \Re^{n_{g} \times n_{g}}, \widehat{\boldsymbol{K}}_{S} \in \Re^{n_{g} \times n_{g}}$, and all their elements $\left(\widehat{\tau}_{s}(z, j), \widehat{\kappa}_{s(z, j)}\right)$ are random variables $\widehat{\tau}_{s(z, j)} \sim N(\mu, \sigma)$, $\widehat{\kappa}_{s(z, j)} \sim N(\mu, \sigma)$ normally distributed with mean $\mu$ and standard deviation $\sigma$.

For the last, we change (10) to

$\partial \boldsymbol{x}=(\boldsymbol{B} \cdot \boldsymbol{x}) \odot(\boldsymbol{M} \cdot(\boldsymbol{x} \oslash \boldsymbol{p}))+\boldsymbol{\Gamma} \cdot \boldsymbol{x}+\boldsymbol{\phi} \odot \widehat{\boldsymbol{\phi}}$,

where $\widehat{\boldsymbol{\phi}} \in \Re^{n_{x}}$ and all their elements $\widehat{\phi}_{s(z, j)}$ are random variables continuous uniformly distributed on the interval $[0,1]$ and $\|\widehat{\boldsymbol{\phi}}\|_{1}=1$.

Example 3: GSEM model to a population stratified into three classes and two groups.

Applying the GSEM model to the population of Fig. 2, we have a situation with three classes $(\mathrm{S}, \mathrm{I}, \mathrm{R})$ and two groups without demography. Thus, $n_{c}=3, n_{g}=2, x \in \mathfrak{R}^{6}$, and we define the matrices

$\boldsymbol{B}=\left[\begin{array}{ccc}\mathbf{0} & -\boldsymbol{B}_{S} & \mathbf{0} \\ \mathbf{0} & \boldsymbol{B}_{S} & \mathbf{0} \\ \mathbf{0} & \mathbf{0} & \mathbf{0}\end{array}\right], \quad \boldsymbol{\Gamma}=\left[\begin{array}{ccc}\mathbf{0} & \mathbf{0} & \mathbf{0} \\ \mathbf{0} & -\boldsymbol{\Gamma}_{i} & \mathbf{0} \\ \mathbf{0} & \boldsymbol{\Gamma}_{i} & \mathbf{0}\end{array}\right], \quad \boldsymbol{M}=\left[\begin{array}{ccc}\boldsymbol{I} & \mathbf{0} & \mathbf{0} \\ \boldsymbol{I} & \mathbf{0} & \mathbf{0} \\ \mathbf{0} & \mathbf{0} & \mathbf{0}\end{array}\right]$,

based on $\boldsymbol{B}_{S}, \boldsymbol{\Gamma}_{i}$, respectively, where $\mathbf{0} \in \mathfrak{R}^{n_{g} \times n_{g}}$ is a null matrix, and $\boldsymbol{I} \in \mathfrak{R}^{n_{g} \times n_{g}}$ is the identity matrix. We consider the vectors $\left(\boldsymbol{s}, \boldsymbol{i}, \boldsymbol{r}, \boldsymbol{p}_{g}, \boldsymbol{\phi}_{s}, \boldsymbol{\phi}_{i}, \boldsymbol{\phi}_{r}\right)$ in the definition of

$\boldsymbol{x}=\left[\begin{array}{l}\boldsymbol{x}_{1} \\ \boldsymbol{x}_{2} \\ \boldsymbol{x}_{3}\end{array}\right]=\left[\begin{array}{l}\boldsymbol{s} \\ \boldsymbol{i} \\ \boldsymbol{r}\end{array}\right], \quad \boldsymbol{p}=\left[\begin{array}{l}\boldsymbol{p}_{g} \\ \boldsymbol{p}_{g} \\ \boldsymbol{p}_{g}\end{array}\right], \quad \boldsymbol{\phi}=\left[\begin{array}{l}\boldsymbol{\phi}_{s} \\ \boldsymbol{\phi}_{i} \\ \boldsymbol{\phi}_{r}\end{array}\right]$.

Example 4: GSEM model to a population stratified into four classes and two groups.

Applying the GSEM model to the population of Fig. 3, we have a situation with four classes $(\mathrm{S}, \mathrm{E}, \mathrm{I}, \mathrm{R})$ and two groups without demography. Thus, $n_{c}=4, n_{g}=2, \boldsymbol{x} \in \mathfrak{R}^{8}$, and we define the matrices

$$
\boldsymbol{B}=\left[\begin{array}{cccc}
\mathbf{0} & \mathbf{0} & -\boldsymbol{B}_{S} & \mathbf{0} \\
\mathbf{0} & \mathbf{0} & \boldsymbol{B}_{S} & \mathbf{0} \\
\mathbf{0} & \mathbf{0} & \mathbf{0} & \mathbf{0} \\
\mathbf{0} & \mathbf{0} & \mathbf{0} & \mathbf{0}
\end{array}\right], \quad \boldsymbol{\Gamma}=\left[\begin{array}{cccc}
\mathbf{0} & \mathbf{0} & \mathbf{0} & \mathbf{0} \\
\mathbf{0} & -\boldsymbol{\Gamma}_{e} & \mathbf{0} & \mathbf{0} \\
\mathbf{0} & \boldsymbol{\Gamma}_{e} & -\boldsymbol{\Gamma}_{i} & \mathbf{0} \\
\mathbf{0} & \mathbf{0} & \boldsymbol{\Gamma}_{i} & \mathbf{0}
\end{array}\right],
$$

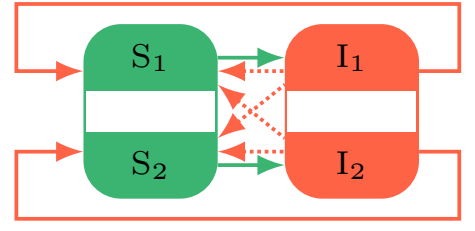

Fig. 4 Flowchart of the GSEM model applied to a population stratified into two classes and two groups without flux between groups and demography

$M=\left[\begin{array}{llll}I & 0 & 0 & 0 \\ I & 0 & 0 & 0 \\ 0 & 0 & 0 & 0 \\ 0 & 0 & 0 & 0\end{array}\right]$

based on $\boldsymbol{B}_{s}, \boldsymbol{\Gamma}_{e}, \boldsymbol{\Gamma}_{i}$. We consider the vectors (s, $\boldsymbol{e}, \boldsymbol{i}, \boldsymbol{r}, \boldsymbol{p}_{g}$, $\left.\boldsymbol{\phi}_{s}, \boldsymbol{\phi}_{e}, \boldsymbol{\phi}_{i}, \boldsymbol{\phi}_{r}\right)$ in the definition of

$\boldsymbol{x}=\left[\begin{array}{l}\boldsymbol{x}_{1} \\ \boldsymbol{x}_{2} \\ \boldsymbol{x}_{3} \\ \boldsymbol{x}_{4}\end{array}\right]=\left[\begin{array}{l}\boldsymbol{s} \\ \boldsymbol{e} \\ \boldsymbol{i} \\ \boldsymbol{r}\end{array}\right], \quad \boldsymbol{p}=\left[\begin{array}{l}\boldsymbol{p}_{g} \\ \boldsymbol{p}_{g} \\ \boldsymbol{p}_{g} \\ \boldsymbol{p}_{g}\end{array}\right], \quad \boldsymbol{\phi}=\left[\begin{array}{l}\boldsymbol{\phi}_{s} \\ \boldsymbol{\phi}_{e} \\ \boldsymbol{\phi}_{i} \\ \boldsymbol{\phi}_{r}\end{array}\right]$.

Example 5: GSEM model to a population stratified into two classes and two groups.

The following example will describe a disease in that individuals who recover become immediately susceptible again, such as influenza. Here, we use two classes and two groups to represents the SIS Model stratified into groups (Fig. 4).

In the situation of Fig. 4, we do not have demography and flux between the groups. Thus, $n_{c}=2, n_{g}=2, x \in \mathfrak{R}^{4}$, and we define the matrices

$\boldsymbol{B}=\left[\begin{array}{cc}\mathbf{0} & -\boldsymbol{B}_{s} \\ \mathbf{0} & \boldsymbol{B}_{s}\end{array}\right], \quad \boldsymbol{\Gamma}=\left[\begin{array}{cc}\mathbf{0} & \boldsymbol{\Gamma}_{i} \\ \mathbf{0} & -\boldsymbol{\Gamma}_{i}\end{array}\right], \quad \boldsymbol{M}=\left[\begin{array}{ll}\boldsymbol{I} & \mathbf{0} \\ \boldsymbol{I} & \mathbf{0}\end{array}\right]$

based on $\boldsymbol{B}_{S}, \boldsymbol{\Gamma}_{i}$, respectively, where $\mathbf{0} \in \Re^{n_{g} \times n_{g}}$ is a null matrix, and $\boldsymbol{I} \in \mathfrak{R}^{n_{g} \times n_{g}}$ is the identity matrix. We consider the vectors $\left(\boldsymbol{s}, \boldsymbol{i}, \boldsymbol{r}, \boldsymbol{p}_{g}, \boldsymbol{\phi}_{s}, \boldsymbol{\phi}_{i}, \boldsymbol{\phi}_{r}\right)$ in the definition of

$\boldsymbol{x}=\left[\begin{array}{l}\boldsymbol{x}_{1} \\ \boldsymbol{x}_{2}\end{array}\right]=\left[\begin{array}{l}\boldsymbol{s} \\ \boldsymbol{i}\end{array}\right], \quad \boldsymbol{p}=\left[\begin{array}{l}\boldsymbol{p}_{g} \\ \boldsymbol{p}_{g}\end{array}\right], \quad \boldsymbol{\phi}=\left[\begin{array}{l}\mathbf{0} \\ \mathbf{0}\end{array}\right]$.

Example 6: GSEM model to a population stratified into four classes and one group.

In the next example, we will apply the GSEM to describe the model used in the work of Batistela et al. (2021), which is an SIRSi model for the COVID-19 pandemic with immunity loss. In the SIRSi model proposed by the authors, they divide the individuals into four classes, S, I, K, R, according to Fig. 5. Class $\mathrm{K}$ is to the individuals with severe symptoms seeking medical attention.

Applying the GSEM model to describe the SIRSi model, we have four classes $(\mathrm{S}, \mathrm{I}, \mathrm{K}, \mathrm{R})$ and one group with demog- 


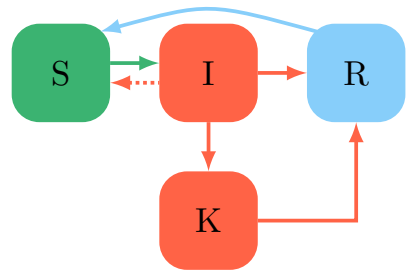

Fig. 5 Flowchart of the GSEM model applied to a population stratified into four classes and one group with demography

raphy. Thus, $n_{c}=4, n_{g}=1, \boldsymbol{x} \in \Re^{4}$, and we define the matrices

$\boldsymbol{B}=\left[\begin{array}{cccc}\mathbf{0} & -\boldsymbol{B}_{s} & \mathbf{0} & 0 \\ \mathbf{0} & \boldsymbol{B}_{s} & \mathbf{0} & \mathbf{0} \\ \mathbf{0} & \mathbf{0} & \mathbf{0} & \mathbf{0} \\ \mathbf{0} & \mathbf{0} & \mathbf{0} & \mathbf{0}\end{array}\right], \quad \boldsymbol{\Gamma}=\left[\begin{array}{cccc}\mathbf{0} & \mathbf{0} & \mathbf{0} & \boldsymbol{\Gamma}_{r} \\ \mathbf{0} & -\boldsymbol{\Gamma}_{i 1}-\boldsymbol{\Gamma}_{i 2} & \mathbf{0} & \mathbf{0} \\ \mathbf{0} & \boldsymbol{\Gamma}_{i 1} & -\boldsymbol{\Gamma}_{k} & \mathbf{0} \\ \mathbf{0} & \boldsymbol{\Gamma}_{i 2} & \boldsymbol{\Gamma}_{k} & -\boldsymbol{\Gamma}_{r}\end{array}\right]$,

$M=\left[\begin{array}{llll}I & 0 & 0 & 0 \\ I & 0 & 0 & 0 \\ 0 & 0 & 0 & 0 \\ 0 & 0 & 0 & 0\end{array}\right]$

based on $\boldsymbol{B}_{s}, \boldsymbol{\Gamma}_{e}, \boldsymbol{\Gamma}_{i}$. We consider the vectors $\left(\boldsymbol{s}, \boldsymbol{i}, \boldsymbol{k}, \boldsymbol{r}, \boldsymbol{p}_{g}\right.$, $\left.\boldsymbol{\phi}_{s}, \boldsymbol{\phi}_{i}, \boldsymbol{\phi}_{k}, \boldsymbol{\phi}_{r}\right)$ in the definition of

$\boldsymbol{x}=\left[\begin{array}{l}\boldsymbol{x}_{1} \\ \boldsymbol{x}_{2} \\ \boldsymbol{x}_{3} \\ \boldsymbol{x}_{4}\end{array}\right]=\left[\begin{array}{l}\boldsymbol{s} \\ \boldsymbol{i} \\ \boldsymbol{k} \\ \boldsymbol{r}\end{array}\right], \quad \boldsymbol{p}=\left[\begin{array}{l}\boldsymbol{p}_{g} \\ \boldsymbol{p}_{g} \\ \boldsymbol{p}_{g} \\ \boldsymbol{p}_{g}\end{array}\right], \quad \boldsymbol{\phi}=\left[\begin{array}{c}\boldsymbol{\phi}_{s} \\ \boldsymbol{\phi}_{i} \\ \boldsymbol{\phi}_{k} \\ \boldsymbol{\phi}_{r}\end{array}\right]$.

\section{Numerical Simulations}

In this section, we present the numerical simulation results and analyze them. We use the GSEM model for all simulations, being (10) for deterministic and (16) for stochastic. We use the Euler method as the first-order numerical procedure for solving ordinary differential equations (ODEs), and we are using steps of 1 day size. All simulations have 300 days, a population of one million, recovery rate $\gamma_{i}=0.2$, and the initial condition is one infected individual in group 1 with the rest of the individuals being susceptible.

To the stochastic case, consider $\widehat{\tau}_{s(z, j)}=0, \widehat{\kappa}_{s(z, j)} \sim$ $N(\mu=0, \sigma=0.5)$ as a random variables normally distributed with null mean and standard deviation 0.5 , and $\|\widehat{\boldsymbol{\phi}}\|_{1}=1$ with your elements being random variables continuous uniformly distributed on the interval $[0,1]$.

The values of $\boldsymbol{T}_{s}, \boldsymbol{K}_{s}, \boldsymbol{\Gamma}_{i}, \boldsymbol{\Lambda}_{s, i, r}$, and $\boldsymbol{\Gamma}_{i}$ are a merge of the parameters' values of the works Mishra et al. (2020); Cotta et al. (2020); Batistela et al. (2021).

About the simulation results, the lines represent the dynamic of infected individuals for the deterministic case, and the filled region shows the dynamic of infected indi-
Fig. 6 Group relationship flowchart of the GSEM model applied to a population stratified into two groups with daily contact and flux between the groups and without demography

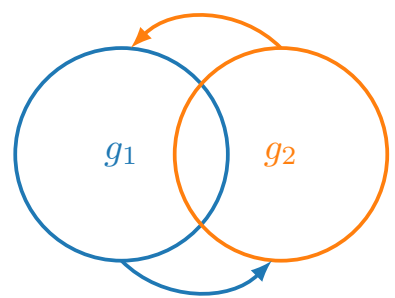

viduals for the stochastics case. The filled region up and down limits, corresponding to maximum and minimum values obtained in four simulations of the same scenario.

Figure 6 shows a flowchart that describes the relationship between two groups. In this flowchart, we use circles with population size proportional to the radius to define each group. To represent the relationship between the groups, we use arrows and the group intersection. The arrows exhibit the existing flux, and the intersection reports the percentile of the daily contact between groups of individuals. For example, the flowchart of Fig. 6 can represent two cities in a metropolitan region that have daily contact between a part of their population.

We divide this section into seven subsections, in which, except the discussion subsection, show a specific case simulation. We simulated examples 3, 4, and 5 in Sects. 4.1, 4.3, and 4.4, respectively. We simulated the GSEM model considering $\boldsymbol{K}_{s}$ as a time-variant matrix in Sects. 4.1 and 4.6 . In Sect. 4.5, we demonstrated a new case, and in Sect. 4.8, we discuss the results.

\subsection{GSEM Model to Three Classes and Two Groups}

In the first simulation, we have one million population divided into two groups with the same number of individuals. According to Example 3, to this simulation, we define the GSEM model parameters as

$$
\begin{aligned}
\boldsymbol{T}_{s} & =\left[\begin{array}{ll}
0.1 & 0.1 \\
0.1 & 0.1
\end{array}\right], & \boldsymbol{K}_{s}=\left[\begin{array}{cc}
4.0 & 0.01 \\
0.01 & 2.5
\end{array}\right], \\
\boldsymbol{\Gamma}_{i} & =\left[\begin{array}{cc}
0.2 & 0 \\
0 & 0.2
\end{array}\right], & \boldsymbol{\Lambda}_{s, i, r}=\left[\begin{array}{cc}
0 & 0.0001 \\
-0.0001 & 0
\end{array}\right] .
\end{aligned}
$$

In Figs. 7 and 8, we can see the behavior of infected individuals is strongly related to their group characteristics, which occurs due to the small flux and small daily contacts percentile between the groups. Although the simulation starts with just one infected individual in group 1 , the disease spread to both groups. The contact between the groups is responsible for the epidemic outbreak in both groups.

\subsection{GSEM Model to Three Classes and Two Groups with a $K_{s}$ Time-Variant Matrix}

In the second simulation, we demonstrate the effects of timevariant parameters to describe the population reactions. Here 


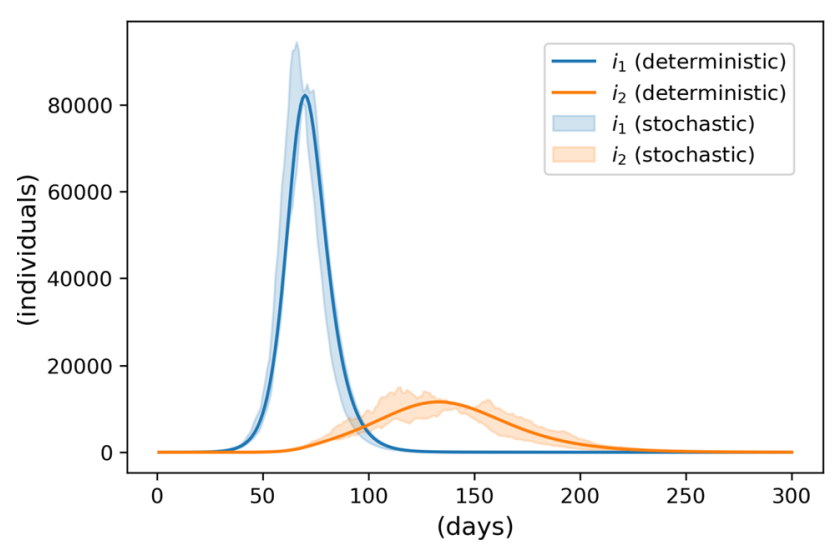

Fig. 7 GSEM model to three classes and two groups, where the blue and orange lines are the infected individuals of groups 1 and 2, respectively. And the light blue and light orange regions indicate the range of infected individuals of groups 1 and 2, respectively, when we consider the stochastic effects (Color figure online)

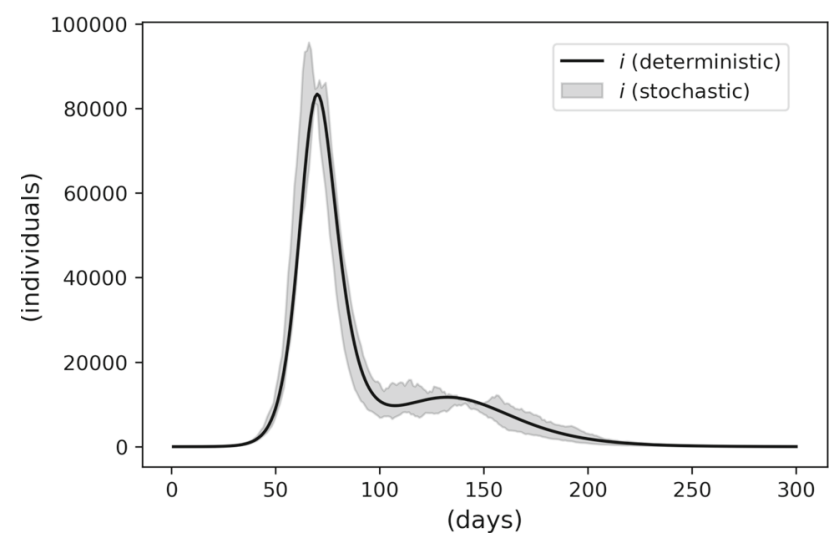

Fig. 8 GSEM model to three classes and two groups, where the black line is the infected individuals of all groups together, and the light gray region indicate the range of infected individuals of all groups together when we consider the stochastic effects

we change $\boldsymbol{K}_{S}$ to

$\boldsymbol{K}_{s}=\left[\begin{array}{cc}4.0 & 0.01 \\ 0.01 & 2.5\end{array}\right]+\left[\begin{array}{cc}\kappa_{x(1,1)}(t) & 0 \\ 0 & \kappa_{x(2,2)}(t)\end{array}\right]$,

where

$\kappa_{x(1,1)}(t)=1.5 \sin \left(\frac{2 \pi t}{200}\right)+0.5 \sin \left(\frac{2 \pi t}{7}\right)$

and

$\kappa_{x(2,2)}(t)=1.0 \sin \left(\frac{2 \pi t}{50}\right)+0.25 \sin \left(\frac{2 \pi t}{14}\right)$.

We choose (17), (18) as a sum of sinusoidal signals to represent the weekly, biweekly and seasonal effect in the population reactions.

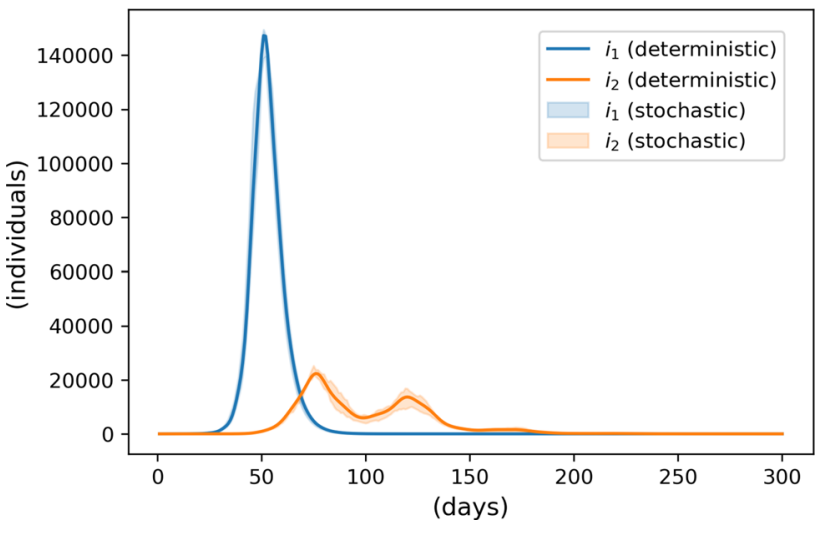

Fig. 9 GSEM model to three classes and two groups, where the blue and orange lines are the infected individuals of groups 1 and 2, respectively. And the light blue and light orange regions indicate the range of infected individuals of groups 1 and 2, respectively, when we consider the stochastic effects (Color figure online)

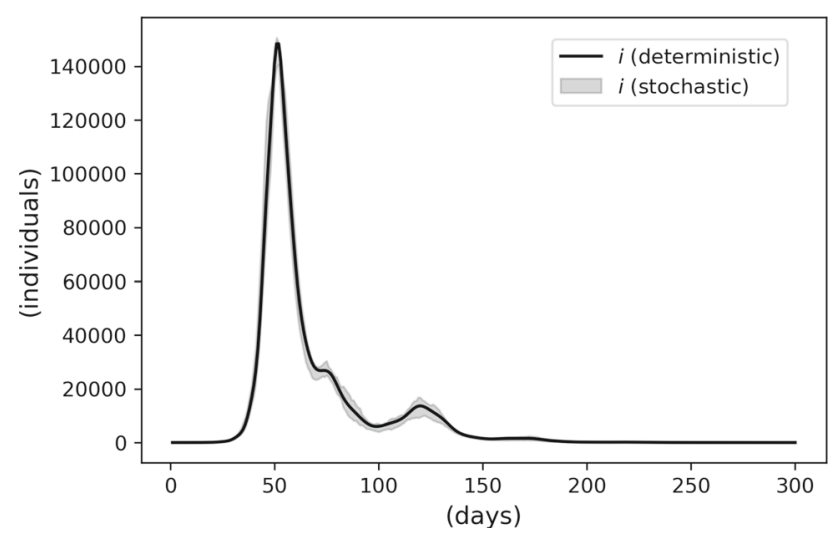

Fig. 10 GSEM model to three classes and two groups, where the black line is the infected individuals of all groups together, and the light gray region indicate the range of infected individuals of all groups together when we consider the stochastic effects

In Figs. 9 and 10, we see the population reactions affecting the behavior of infected individuals. Depending on the reaction, the peak of infected individuals can grow or can appear multiples peaks.

In the third simulation, we change, again, the value of $\boldsymbol{K}_{S}$, now to

$\boldsymbol{K}_{s}=\left[\begin{array}{cc}2.1 & 0.01 \\ 0.01 & 2.5\end{array}\right]+\left[\begin{array}{cc}\kappa_{x(1,1)}(t) & 0 \\ 0 & \kappa_{x(2,2)}(t)\end{array}\right]$,

and we keep (17), (18) values.

In Figs. 11 and 12, it is more evident the effects of the population reaction. In this simulation, we can see that the last peak, considering the stochastic case, can or not occurs. These results demonstrate that the behavior of an epidemic disease can be very complex. 


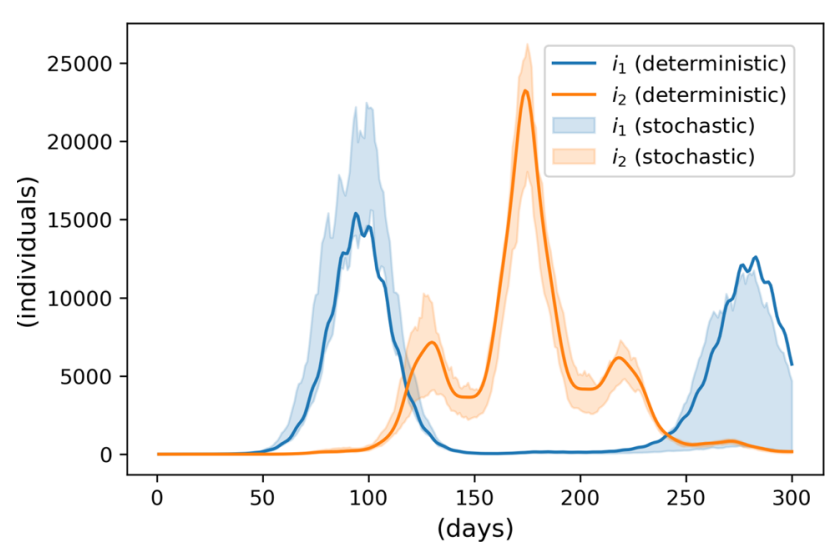

Fig. 11 GSEM model to three classes and two groups, where the blue and orange lines are the infected individuals of groups 1 and 2, respectively. And the light blue and light orange regions indicate the range of infected individuals of groups 1 and 2, respectively, when we consider the stochastic effects (Color figure online)

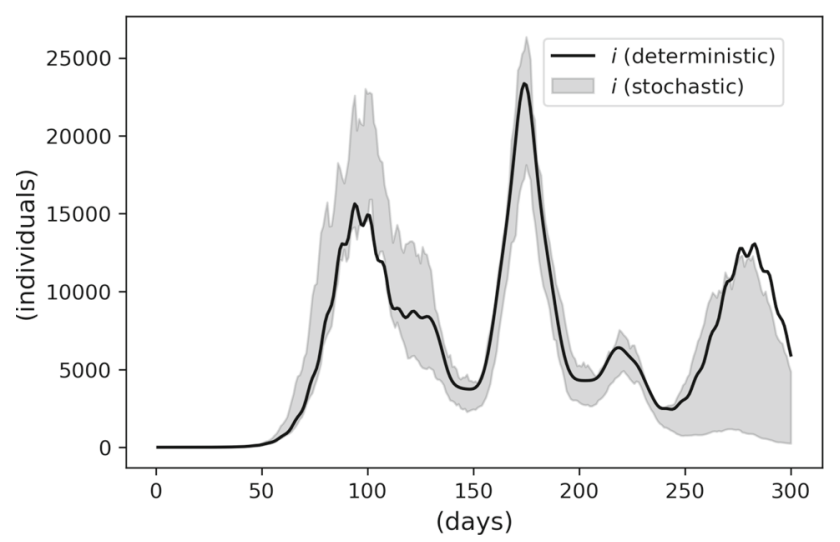

Fig. 12 GSEM model to three classes and two groups, where the black line is the infected individuals of all groups together, and the light gray region indicate the range of infected individuals of all groups together when we consider the stochastic effects

\subsection{GSEM Model to Four Classes and Two Groups}

In the fourth simulation, we have one million population divided into two groups with the same number of individuals. The GSEM model parameters are the same as Sect. 4.1, adding a latency period of 5 days, which results in

$\boldsymbol{\Gamma}_{e}=\left[\begin{array}{cc}0.2 & 0 \\ 0 & 0.2\end{array}\right]$

In Figs. 13 and 14 , we can see, again, the behavior of infected individuals is strongly related to their group characteristics, which occurs due to the small flux and small daily contacts percentile between the groups. The latency period makes the peak of infected individuals slower and smaller when compared to the result of Sect. 4.1. Again, the epidemic disease spread through the groups.

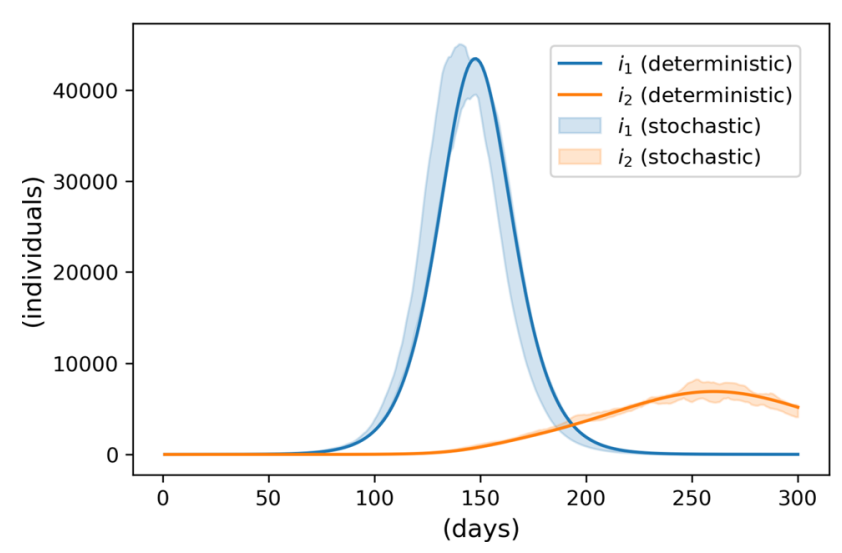

Fig. 13 GSEM model to four classes and two groups, where the blue and orange lines are the infected individuals of groups 1 and 2, respectively. And the light blue and light orange regions indicate the range of infected individuals of groups 1 and 2, respectively, when we consider the stochastic effects (Color figure online)

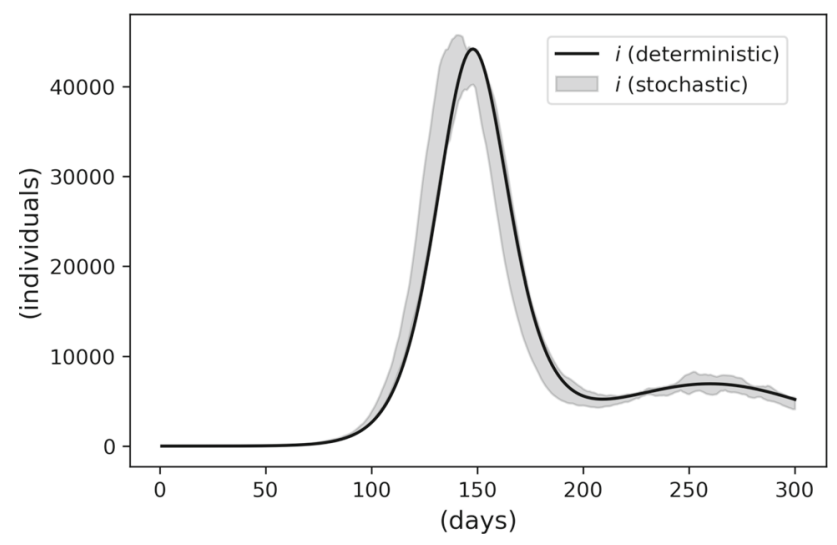

Fig. 14 GSEM model to four classes and two groups, where the black line is the infected individuals of all groups together, and the light gray region indicate the range of infected individuals of all groups together when we consider the stochastic effects

\subsection{GSEM Model to Two Classes and Two Groups}

In the fifth simulation, we have one million population divided into two groups with the same number of individuals. According to Example 5, to this simulation, we define the GSEM model parameters as

$$
\begin{aligned}
\boldsymbol{T}_{s}=\left[\begin{array}{ll}
0.1 & 0.1 \\
0.1 & 0.1
\end{array}\right], & \boldsymbol{K}_{s}=\left[\begin{array}{cc}
4.0 & 0.01 \\
0.01 & 2.5
\end{array}\right], \\
\boldsymbol{\Gamma}_{i}=\left[\begin{array}{cc}
0.2 & 0 \\
0 & 0.2
\end{array}\right], & \boldsymbol{\Lambda}_{s, i, r}=\left[\begin{array}{ll}
0 & 0 \\
0 & 0
\end{array}\right] .
\end{aligned}
$$

In Figs. 15 and 16 , we can see the behavior of infected individuals converging to the endemic equilibrium. In this simulation, we did not use the flux to guarantee that each group, after a time, will go to your endemic equilibrium. Although the simulation starts with just one infected individ- 


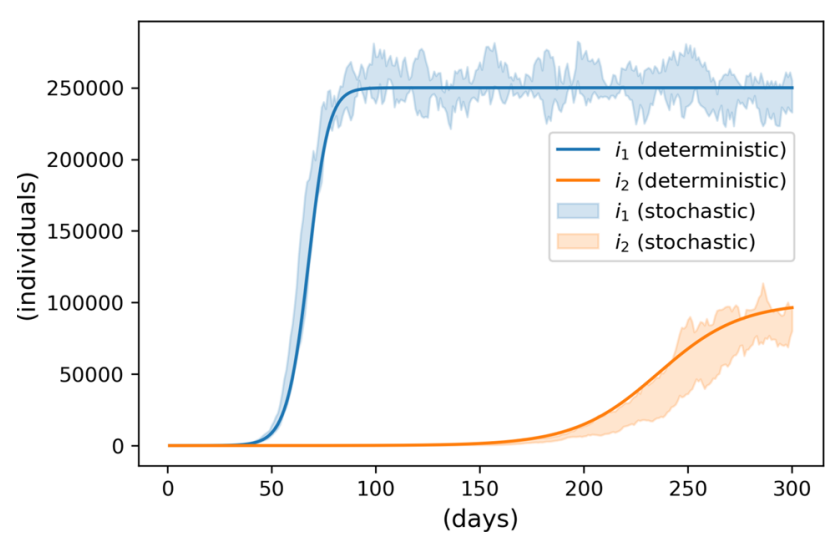

Fig. 15 GSEM model to three classes and two groups, where the blue and orange lines are the infected individuals of groups 1 and 2, respectively. And the light blue and light orange regions indicate the range of infected individuals of groups 1 and 2, respectively, when we consider the stochastic effects (Color figure online)

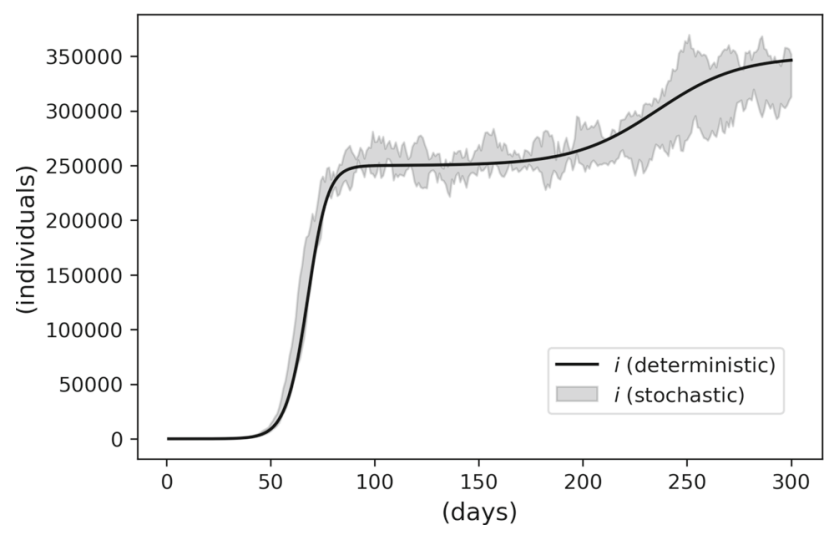

Fig. 16 GSEM model to three classes and two groups, where the black line is the infected individuals of all groups together, and the light gray region indicate the range of infected individuals of all groups together when we consider the stochastic effects

ual in group 1, the disease spread to both groups. The daily contact between the groups is responsible for the epidemic outbreak in both groups.

\subsection{GSEM Model to Three Classes and Eight Groups}

The flowchart of Fig. 17 describes the relationship of a population divided into eight groups. Just two of them have daily contact, and the arrows indicate how flux occurs. To one million population, we distribute the individuals as follows: $g_{1}=30 \%, g_{2}=g_{3}=15 \%$ and the others $8 \%$ of the population.

In the sixth simulation, we structure the GSEM model into three classes and eight groups. The three classes are biologically similar to the SIR model. The parameters used in this simulation are

$$
\boldsymbol{T}_{s}=0,1 \cdot \mathbf{1}_{8 \times 8}, \quad \boldsymbol{\Gamma}_{i}=0,2 \cdot \boldsymbol{I},
$$

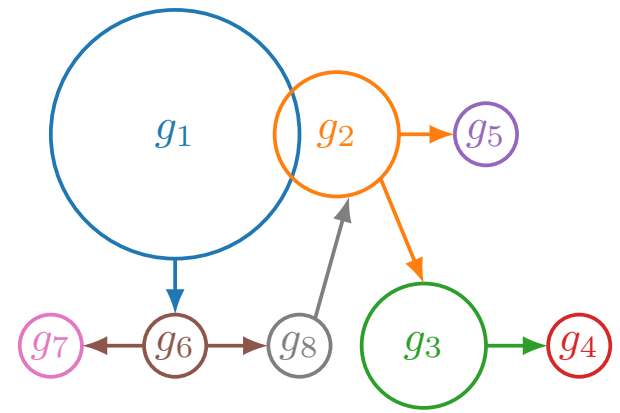

Fig. 17 Group relationship flowchart of the GSEM model applied to a population stratified into eight groups with daily contact between two groups, the flux between all groups, and without demography

$$
\begin{gathered}
\boldsymbol{K}_{s}=\left[\begin{array}{cccccccc}
4.0 & 0.009 & 0 & 0 & 0 & 0 & 0 & 0 \\
0.009 & 2.5 & 0 & 0 & 0 & 0 & 0 & 0 \\
0 & 0 & 2.4 & 0 & 0 & 0 & 0 & 0 \\
0 & 0 & 0 & 3.0 & 0 & 0 & 0 & 0 \\
0 & 0 & 0 & 0 & 2.6 & 0 & 0 & 0 \\
0 & 0 & 0 & 0 & 0 & 4.5 & 0 & 0 \\
0 & 0 & 0 & 0 & 0 & 0 & 2.8 & 0 \\
0 & 0 & 0 & 0 & 0 & 0 & 0 & 3.1
\end{array}\right], \\
\boldsymbol{\Lambda}_{s, i, r}=0.002 \cdot\left[\begin{array}{ccccccccc}
0 & 0 & 0 & 0 & 0 & -1 & 0 & 0 \\
0 & 0 & -1 & 0 & -1.5 & 0 & 0 & 3 \\
0 & 1 & 0 & -1 & 0 & 0 & 0 & 0 \\
0 & 0 & 1 & 0 & 0 & 0 & 0 & 0 \\
0 & 1.5 & 0 & 0 & 0 & 0 & 0 & 0 \\
1 & 0 & 0 & 0 & 0 & 0 & -2 & -3 \\
0 & 0 & 0 & 0 & 0 & 2 & 0 & 0 \\
0 & -3 & 0 & 0 & 0 & 3 & 0 & 0
\end{array}\right] .
\end{gathered}
$$

To the stochastic case, consider $\widehat{\kappa}_{s(z, j)} \sim N(\mu=0, \sigma=$ $0.5)$, and $\|\widehat{\phi}\|_{1}=1$. In this simulation, all groups use individual's mean values of $R_{0}$ within the range of 1.2 to 2.25 .

In Figs. 18 and 19 , we can see the behavior of infected individuals together and separated by groups. Based on this simulation, we can conclude, the population organization is crucial to an epidemic model. To determine how the epidemic disease will spread, it is necessary good knowledge about the population. Although the simulation starts with just one infected individual in group 1, the disease spread to all groups.

Another aspect we highlight is the low influence of stochastic effects. The groups' relationship shows more relevant in the final result than random variables. This effect does not surprise us. According to the literature, the stochastic effects are more influential in small populations (Daley and Gani 1999). 


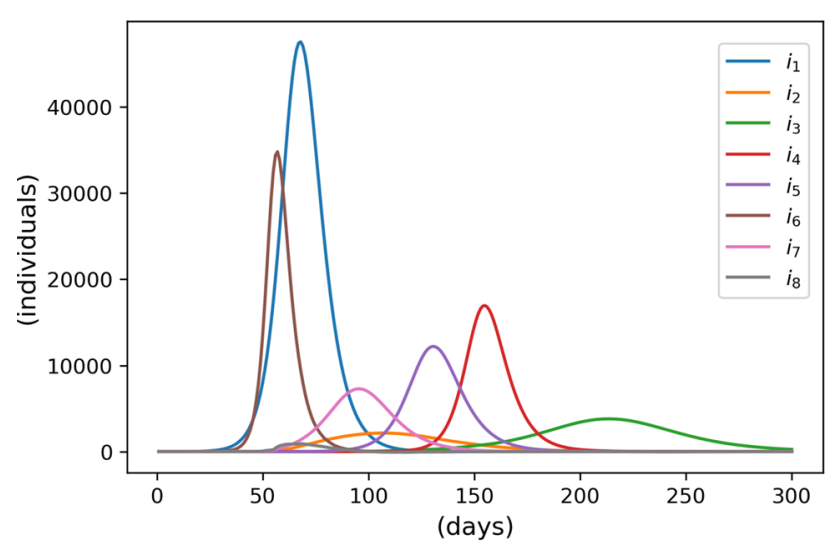

Fig. 18 GSEM model to three classes and eight groups, where each colored line represents the infected individuals of a specific group (Color figure online)

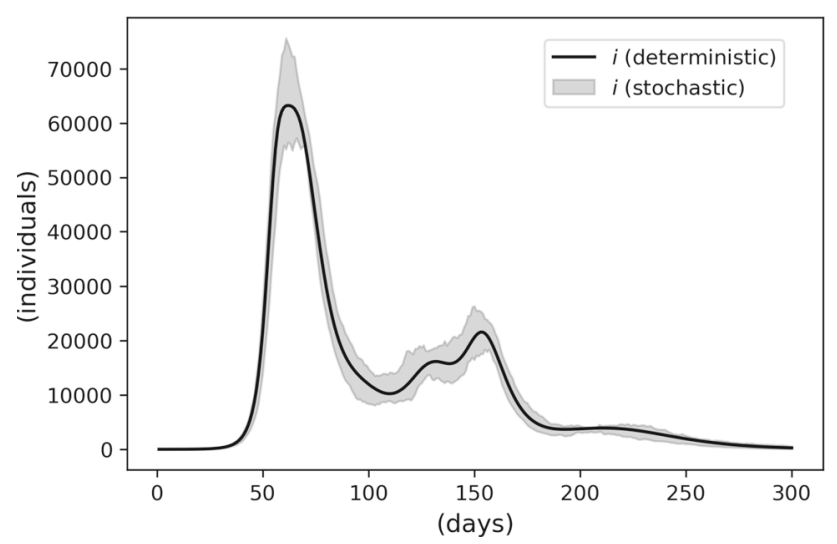

Fig. 19 GSEM model to three classes and eight groups, where the black line is the infected individuals of all groups together, and the light gray region indicate the range of infected individuals of all groups together when we consider the stochastic effects

\subsection{GSEM Model to Three Classes and Eight Groups with a $K_{s}$ Time-Variant Matrix}

In the seventh simulation, one more time, we will evaluate the behavior of infected individuals when $\boldsymbol{K}_{S}$ is time variant. Thus, we keep the same adjust of Sect. 4.5, and we add to each element of the main diagonal of $\boldsymbol{K}_{S}$ one of these elements

$$
\begin{aligned}
& \kappa_{x}(1,1)(t)=1.5 \sin \left(\frac{2 \pi t}{200}\right)+0.5 \sin \left(\frac{2 \pi t}{7}\right), \\
& \kappa_{x}(2,2)(t)=1.0 \sin \left(\frac{2 \pi t}{50}\right)+0.25 \sin \left(\frac{2 \pi t}{14}\right), \\
& \kappa_{x}(3,3)(t)=0.5 \sin \left(\frac{2 \pi t}{500}\right)+0.5 \sin \left(\frac{2 \pi t}{7}\right), \\
& \kappa_{x}(4,4)(t)=1.5 \sin \left(\frac{2 \pi t}{100}\right)+0.5 \sin \left(\frac{2 \pi t}{7}\right), \\
& \kappa_{x}(5,5)(t)=1.5 \sin \left(\frac{2 \pi t}{300}\right)+1.0 \sin \left(\frac{2 \pi t}{7}\right),
\end{aligned}
$$

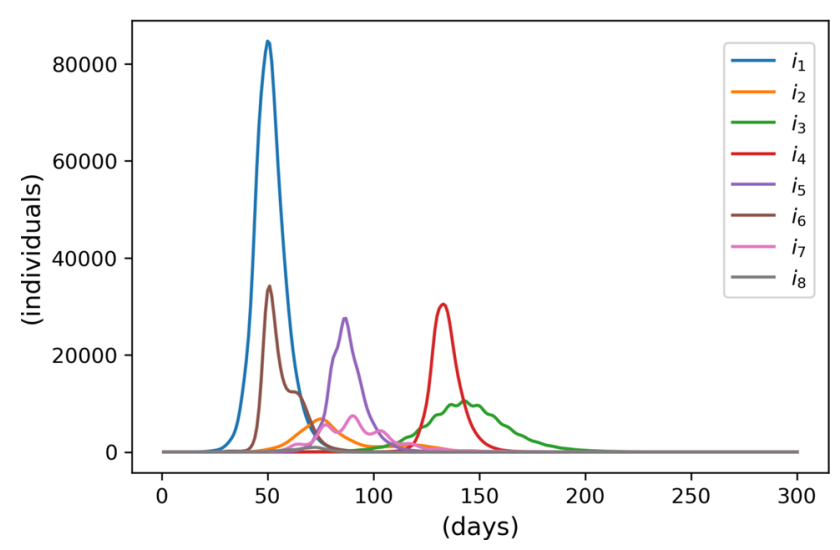

Fig. 20 GSEM model to three classes and eight groups, where each colored line represents the infected individuals of a specific group (Color figure online)

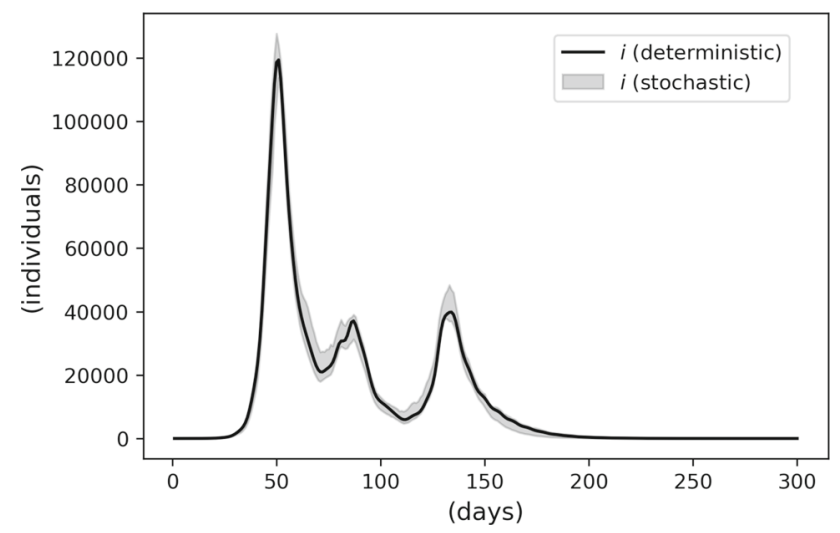

Fig. 21 GSEM model to three classes and eight groups, where the black line is the infected individuals of all groups together, and the light gray region indicate the range of infected individuals of all groups together when we consider the stochastic effects

$\kappa_{x}(6,6)(t)=3.5 \sin \left(\frac{2 \pi t}{20}\right)$,

$\kappa_{x}(7,7)(t)=0.5 \sin \left(\frac{2 \pi t}{150}\right)+1.5 \sin \left(\frac{2 \pi t}{14}\right)$,

$\kappa_{x}(8,8)(t)=2.0 \sin \left(\frac{2 \pi t}{30}\right)+0.5 \sin \left(\frac{2 \pi t}{7}\right)$.

In Figs. 20 and 21 , we can see, one more time, that the population reaction implies the behavior of infected individuals. Thus, we can see that the GSEM model can describe complex behavior, and because of this, we believe that it will be a good option for the researchers and decision-makers.

\subsection{GSEM Model to Four Classes and One Group}

In the eighth simulation, we apply the GSEM to simulate a newer SIRSi model recently proposed by Batistela et al. (2021). We use this simulation to demonstrate the versatility of the GSEM model. Here, we will present that the GSEM 


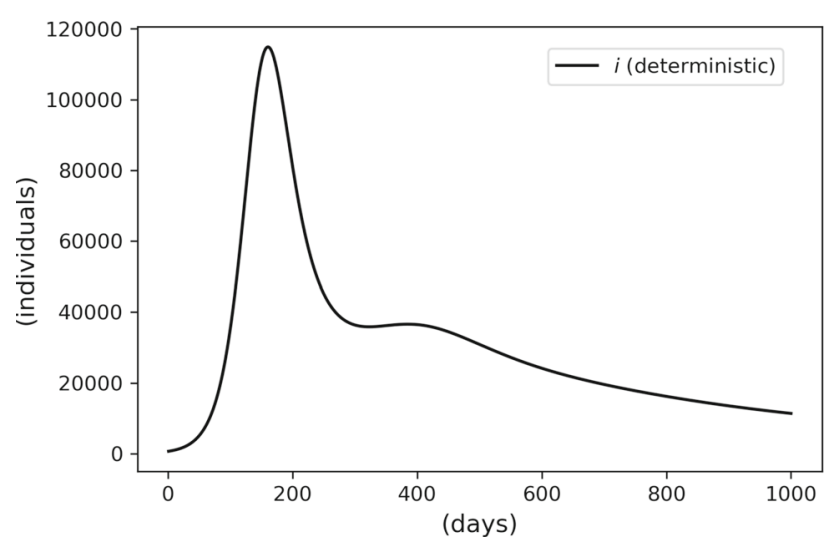

Fig. 22 GSEM model to four classes and one group, where the black line is the infected individuals

can describe other compartmental models applied to a real case.

Then, this simulation is according to Example 6, and we use the parameters of Batistela's work. Thus, we use the deterministic version of the GSEM, Euler method as the first-order numerical procedure for solving ordinary differential equations (ODEs), and we are using steps of 1 day size. This simulation has 1000 days, a population of 11.869.660 individuals (São Paulo, Brazil), $\boldsymbol{B}_{s}=0.3841$, $\boldsymbol{\Gamma}_{i 1}=0.1181, \boldsymbol{\Gamma}_{i 2}=0.2179, \boldsymbol{\Gamma}_{k}=0.1248, \boldsymbol{\Gamma}_{r}=0.0200$, $\boldsymbol{\phi}_{s}=1.773 \cdot 10^{-5} \boldsymbol{s}, \boldsymbol{\phi}_{i}=0, \boldsymbol{\phi}_{k}=-7.195 \cdot 10^{-2} \boldsymbol{k}, \boldsymbol{\phi}_{r}=$ $-1.822 \cdot 10^{-5} \boldsymbol{r}$, and the initial condition is $\boldsymbol{s}_{0}=9.857 \cdot 10^{-1}$, $\boldsymbol{i}_{0}=5.340 \cdot 10^{-5}, \boldsymbol{k}_{0}=8.425 \cdot 10^{-8}$ and $\boldsymbol{r}_{0}=0$. All parameters and initial conditions computed are normalized with respect to the total population.

In Fig. 22, we can see the GSEM showing the same result as the SIRSi model. The contribution of the GSEM model is that its structure allows describing several different behaviors related to biological and populational aspects. This GSEM's characteristic will make it possible to use it associated with AI to get better results in the follow-up of epidemic outbreaks.

\subsection{Discussion}

The simulations of Figs. 7, 8, 9, 10, 11, 12, 13, 14, 15, 16, $17,18,19,20,21$, and 22 are initials results for the proposed GSEM model. We develop them to be didactic, that is, to make it easy to understand the GSEM model, approximating it to the SIR-type models. Be didactic was the reason the majority of simulation uses constant parameters. Using the GSEM model, we can divide the population into groups to stratify it, achieving better contact network representations of the population. In the GSEM model, there are no limits to the number of groups and classes.

The proposed model is versatile, and we can use it to simulate the compartmental models. For example, we can simulate
SIR, SIS, SIRD ${ }^{2}$, MSIR $^{3}$, SEIR, SEIS, MSEIR ${ }^{3}$, MSEIRS $^{3}$, models with vaccination, vector-borne models, multi-strain models, spatial models, models that consider the population density, among others. For each cited model, we can include demography aspects, and we can stratify the population into any number of groups. Another attribute we can highlight in the GSEM model is your compact form, which helps in the development of new strategies of control and parameters estimator.

In the COVID-19 outbreak, we can observe the influence, in the spreading of the disease, of the spatial effects, and the populational density. Thus, it is fundamental to use a model capable to stratify the population according to these aspects. In pandemic events, the disease shows different behaviors to metropolitan regions and small rural cities. Beyond this, other fundamental characteristics, as the etheric pyramid, presents significant differences from location to location. A good compartmental model for the pandemic disease spreading in a population has to incorporate the majority of biological and social aspects. The proposed GSEM model allows to include these aspects in a compact form.

Another consideration is about the time-varying parameters. To describe complex epidemic behavior, we can use models just with the main relationships associated with very complex time-varying parameters or use models with a better and more detailed relationships' description associated with non-complex time-varying parameters. The GSEM uses the second option. However, to do this, the GSEM has to describe all possible relationships. Then, we introduced the flux concept, which produces indirect effects on epidemic diseases, and because of this, the researchers generally ignored it. The flux concept adds to GSEM the possibility to anticipate the changes in epidemics behavior. The flux also allows deterministic models to represent, adequately, the concepts of complex networks.

At last, the GSEM has a set of building rules. This characteristic is important because we can use the GSEM in the next generation of epidemic models. In the future, we will use AI techniques to define the best epidemic model for a specific outbreak. However, the resulting model has to make sense for the researchers, and they have to comprehend it. Thus, when AI techniques use the GSEM building rules, the model produced will be comprehensible.

To summarize, our key benefits are the following:

- We develop a new mixed compartmental epidemic model for heterogeneous populations. This model is called the GSEM (group-structured-epidemic model), and it

\footnotetext{
$\overline{2}$ The letter D represents the cumulative number of individuals that deceased due to the disease.

3 The letter M represents the individuals with maternally derived immunity.
} 
divides the population into homogeneous groups to achieve a better description of the population.

- The GSEM model is versatile, and we can adapt it to several kinds of epidemic diseases. It also is compact, considers demographic and environmental variability, and has no limits to the number of groups or classes.

- The GSEM model can incorporate stochastic effects and time-variant parameters to describe the population reactions.

- The GSEM model introduces the flux concept, which expands the results to the deterministic models.

- The GSEM model is a schematic to work as a set of rules to help AI techniques describe any epidemic outbreak through the compartmental model.

\section{Conclusions}

During the outbreak of COVID-19, the researchers identified the need to adjust the existing models to get better results in simulations of the disease dynamics. Thus, this work proposed a mixed compartmental epidemic model. This model consists of a deterministic model that considers stochastic effects existing in the population and divides the epidemic disease into compartments.

The proposed model, called GSEM, uses two qualifiers (classes and groups) to define each compartment. The GSEM model can have any compartment numbers. In other words, it can have any number of classes or groups. The model describes the biological and populational aspects through the classes and groups, respectively.

The proposed model is versatile, compact, considers demographic and environmental variability, and admits timevariant parameters to describe the population reactions. From the GSEM model, it will be possible to develop new strategies of control and parameters estimator.

Simulation results show the GSEM model for several kinds of epidemic diseases. In some of them, the parameters were constants. To the others, they were time variants. The GSEM simulates epidemic diseases with and without latency period, with two or more groups, purely deterministic or considering stochastic effects, and to a real case situation.

We believe the proposed GSEM model is easy to comprehend, and we can use it to simulate complex scenarios to epidemic diseases. Thus, we consider that GSEM can help in future epidemic diseases or even in future pandemics diseases.

As the next step, we pretend to analyze the mathematical properties of the GSEM model. We will study the model positivity, its equilibrium points, your stability, and your basic reproduction matrix.
Acknowledgements An early version of paper was presented at XXIII Congresso Brasileiro de Automática (CBA 2020).

\section{Declarations}

Conflicts of interest The authors declare that they have no known competing financial interests or personal relationships that could have appeared to influence the work reported in this paper.

\section{References}

Acemoglu, D., Chernozhukov, V., Werning, I., \& Whinston, M.D. (2020). Optimal targeted lockdowns in a multi-group SIR model. Working Paper 27102, National Bureau of Economic Research. https://doi.org/10.3386/w27102.

Allen, L. J. (2017). A primer on stochastic epidemic models: Formulation, numerical simulation, and analysis. Infectious Disease Modelling, 2(2), 128-142. https://doi.org/10.1016/j.idm.2017.03. 001.

Allen, L. J. S., \& Burgin, A. M. (2000). Comparison of deterministic and stochastic SIS and SIR models in discrete time. Mathematical Biosciences, 163(1), 1-33. https://doi.org/10.1016/S00255564(99)00047-4.

Artalejo, J. R., Economou, A., \& Lopez-Herrero, M. J. (2013). Stochastic epidemic models with random environment: Quasi-stationarity, extinction and final size. Journal of Mathematical Biology, 67(4), 799-831. https://doi.org/10.1007/s00285-012-0570-5.

Batistela, C. M., Correa, D. P. F., Bueno, Á. M., \& Piqueira, J. R. C. (2021). SIRSi compartmental model for COVID-19 pandemic with immunity loss. Chaos, Solitons and Fractals, 142, 110388. https:// doi.org/10.1016/j.chaos.2020.110388.

Bedford, J., Farrar, J., Ihekweazu, C., Kang, G., Koopmans, M., \& Nkengasong, J. (2019). A new twenty-first century science for effective epidemic response. Nature, 575(7781), 130-136. https:// doi.org/10.1038/s41586-019-1717-y.

Black, A. J., \& McKane, A. J. (2012). Stochastic formulation of ecological models and their applications. Trends in Ecology and Evolution, 27(6), 337-345. https://doi.org/10.1016/j.tree.2012.01.014.

Blackwood, J. C., \& Childs, L. M. (2018). An introduction to compartmental modeling for the budding infectious disease modeler. Letters in Biomathematics, 5(1), 195-221. https://doi.org/10. 1080/23737867.2018.1509026.

Britton, T. (2010). Stochastic epidemic models: A survey. Mathematical Biosciences, 225(1), 24-35. https://doi.org/10.1016/j.mbs.2010. 01.006.

Calafiore, G. C., Novara, C., \& Possieri, C. (2020). A time-varying SIRD model for the COVID-19 contagion in Italy. Annual Reviews in Control, 50, 361-372. https://doi.org/10.1016/j.arcontrol.2020. 10.005 .

Canabarro, A., Tenório, E., Martins, R., Martins, L., Brito, S., \& Chaves, R. (2020). Data-driven study of the COVID-19 pandemic via agestructured modelling and prediction of the health system failure in Brazil amid diverse intervention strategies. PLoS ONE, 15(7), 1-13. https://doi.org/10.1371/journal.pone.0236310.

Cotta, R. M., Naveira-Cotta, C. P., \& Magal, P. (2020). Mathematical parameters of the COVID-19 epidemic in Brazil and evaluation of the impact of different public health measures. Biology. https:// doi.org/10.3390/biology9080220.

Daley, D. J., \& Gani, J. (1999). Epidemic Modelling: An Introduction. Cambridge Studies in Mathematical Biology. Cam- 
bridge: Cambridge University Press. https://doi.org/10.1017/ CBO9780511608834.

Davies, N. G., Klepac, P., Liu, Y., et al. (2020). Age-dependent effects in the transmission and control of COVID-19 epidemics. Nature Medicine. https://doi.org/10.1038/s41591-020-0962-9.

Dias, S., Queiroz, K., \& Araujo, A. (2020). Modelo matemático misto estruturado em grupos para doenças epidêmicas. In: Anais do Congresso Brasileiro de Automática 2020. https://doi.org/10.48011/ asba.v2i1.997.

Dias, S., Queiroz, K., \& Araujo, A. (2021a). Controlling epidemic diseases based only on social distancing level. Journal of Control, Automation and Electrical Systems. https://doi.org/10.1007/ s40313-021-00745-6.

Dias, S., Queiroz, K., \& Araujo, A. (2021b). Controlling epidemic diseases based only on social distancing level: General case. ISA Transactions. https://doi.org/10.1016/j.isatra.2021.05.004.

Duan, X. C., Jung, I. H., Li, X. Z., \& Martcheva, M. (2020). Dynamics and optimal control of an age-structured SIRVS epidemic model. Mathematical Methods in the Applied Sciences, 43(7), 4239-4256. https://doi.org/10.1002/mma.6190.

Hilton, J., \& Keeling, M. J. (2019). Incorporating household structure and demography into models of endemic disease. Journal of the Royal Society Interface, 16(157), 20190317. https://doi.org/10. 1098/rsif.2019.0317.

Hong, H. G., \& Li, Y. (2020). Estimation of time-varying reproduction numbers underlying epidemiological processes: A new statistical tool for the COVID-19 pandemic. PLOS ONE, 15(7), 1-15. https:// doi.org/10.1371/journal.pone.0236464.

Jiang, J., \& Zhou, T. (2018). Resource control of epidemic spreading through a multilayer network. Scientific Reports, 8(1), 1629. https://doi.org/10.1038/s41598-018-20105-w.

Kermack, W.O., McKendrick, A.G., \& Walker, G.T. (1927). A contribution to the mathematical theory of epidemics. Proceedings of the Royal Society of London Series A, Containing Papers of a Mathematical and Physical Character 115(772): 700-721. https:// doi.org/10.1098/rspa.1927.0118

Kucharski, A. J., Russell, T. W., Diamond, C., et al. (2020). Early dynamics of transmission and control of COVID-19: a mathematical modelling study. The Lancet Infectious Diseases, 20(5), 553-558. https://doi.org/10.1016/S1473-3099(20)30144-4.

Li, Q., Guan, X., Wu, P., et al. (2020). Early transmission dynamics in Wuhan, China, of novel coronavirus-infected pneumonia. New England Journal of Medicine, 382(13), 1199-1207. https://doi. org/10.1056/NEJMoa2001316.

López-García, M. (2016). Stochastic descriptors in an SIR epidemic model for heterogeneous individuals in small networks. Mathematical Biosciences, 271, 42-61. https://doi.org/10.1016/j.mbs.2015. 10.010 .

Martcheva, M. (2015). An Introduction to Mathematical Epidemiology. Texts in Applied Mathematics. New York: Springer.

McKendrick, A. G. (1925). Applications of mathematics to medical problems. Proceedings of the Edinburgh Mathematical Society. https://doi.org/10.1017/S0013091500034428.

Mishra, B. K., Keshri, A. K., Rao, Y. S., Mishra, B. K., Mahato, B., Ayesha, S., et al. (2020). COVID-19 created chaos across the globe: Three novel quarantine epidemic models. Chaos, Solitons and Fractals. https://doi.org/10.1016/j.chaos.2020.109928.
Moreno, Y., Pastor-Satorras, R., \& Vespignani, A. (2002). Epidemic outbreaks in complex heterogeneous networks. The European Physical Journal B: Condensed Matter and Complex Systems, 26(4), 521-529. https://doi.org/10.1140/epjb/e20020122.

Newman, M. E. J. (2002). Spread of epidemic disease on networks. Physical Review E, 66, 016128. https://doi.org/10.1103/ PhysRevE.66.016128.

Nowzari, C., Preciado, V. M., \& Pappas, G. J. (2016). Analysis and control of epidemics: A survey of spreading processes on complex networks. IEEE Control Systems Magazine, 36(1), 26-46.

Ortega, E. M., Alonso, J., \& Ortega, I. (2013). Stochastic comparisons of mixtures of parametric families in stochastic epidemics. Mathematical Biosciences, 243(1), 18-27. https://doi.org/10.1016/j. mbs.2012.12.006.

Pastor-Satorras, R., Castellano, C., Van Mieghem, P., \& Vespignani, A. (2015). Epidemic processes in complex networks. Review of Modern Physics, 87, 925-979. https://doi.org/10.1103/RevModPhys. 87.925 .

Prem, K., Liu, Y., Russell, T. W., et al. (2020). The effect of control strategies to reduce social mixing on outcomes of the COVID-19 epidemic in Wuhan, China: a modelling study. The Lancet Public Health. https://doi.org/10.1016/S2468-2667(20)30073-6.

Roberts, M., Andreasen, V., Lloyd, A., \& Pellis, L. (2015). Nine challenges for deterministic epidemic models. Epidemics, 10, 4953. https://doi.org/10.1016/j.epidem.2014.09.006, challenges in Modelling Infectious DIsease Dynamics

Sabino, E. C., Buss, L. F., Carvalho, M. P. S., Prete, C. A., Crispim, M. A. E., Fraiji, N. A., et al. (2021). Resurgence of COVID-19 in Manaus, Brazil, despite high seroprevalence. The Lancet, 397(10273), 452455. https://doi.org/10.1016/S0140-6736(21)00183-5.

Wang, P., \& Jia, J. (2019). Stationary distribution of a stochastic SIRD epidemic model of Ebola with double saturated incidence rates and vaccination. Advances in Difference Equations, 1, 433. https://doi. org/10.1186/s13662-019-2352-5.

You, C., Deng, Y., Hu, W., Sun, J., Lin, Q., Zhou, F., et al. (2020). Estimation of the time-varying reproduction number of COVID-19 outbreak in China. International Journal of Hygiene and Environmental Health, 228, 113555. https://doi.org/10.1016/j.ijheh.2020. 113555.

Zakary, O., Rachik, M., \& Elmouki, I. (2017a). A new epidemic modeling approach: Multi-regions discrete-time model with travelblocking vicinity optimal control strategy. Infectious Disease Modelling, 2(3), 304-322. https://doi.org/10.1016/j.idm.2017.06. 003.

Zakary, O., Rachik, M., \& Elmouki, I. (2017b). On the analysis of a multi-regions discrete sir epidemic model: An optimal control approach. International Journal of Dynamics and Control, 5(3), 917-930. https://doi.org/10.1007/s40435-016-0233-2.

Zhang, Z., Wang, H., Wang, C., \& Fang, H. (2015). Modeling epidemics spreading on social contact networks. IEEE Transactions on Emerging Topics in Computing, 3(3), 410-419. https://doi.org/ 10.1109/TETC.2015.2398353.

Publisher's Note Springer Nature remains neutral with regard to jurisdictional claims in published maps and institutional affiliations. 